\title{
ADAPTAÇÕES MORFOFUNCIONAIS APÓS 12 SEMANAS DE TREINAMENTO CONCORRENTE EM HOMENS DE MEIA-IDADE
}

Mara Patrícia Traina Chacon-Mikahil, Universidade Estadual de Campinas - UNICAMP, Campinas, São Paulo - Brasil

Cleiton Augusto Libardi, Universidade Estadual de Campinas - UNICAMP, Campinas, São Paulo - Brasil

Felipe Romano Damas Nogueira, Universidade Estadual de Campinas - UNICAMP, Campinas, São Paulo - Brasil

Felipe Cassaro Vechin, Universidade Estadual de Campinas - UNICAMP, Campinas, São Paulo - Brasil

Thiago Gaudensi Costa, Universidade Estadual de Campinas - UNICAMP, Campinas, São Paulo

- Brasil

Claudinei Ferreira dos Santos, Universidade Estadual de Campinas - UNICAMP, Campinas, São

Paulo - Brasil

Vera Aparecida Madruga, Universidade Estadual de Campinas - UNICAMP, Campinas, São Paulo - Brasil

\section{RESUMO}

O objetivo do estudo foi analisar as adaptações morfofuncionais decorrentes de 12 semanas de treinamento concorrente (TC). Quinze homens saudáveis, sedentários, de meia-idade (48,8 \pm 5,0 anos) participaram deste estudo. Foram separados em: grupo treinamento concorrente (TC, n=8), que realizaram exercícios com pesos seguidos de exercícios de caminhada e corrida; e grupo controle $(\mathrm{GC}, \mathrm{n}=7)$, o qual não realizou exercícios físicos durante o período experimental. Foram realizadas avaliações antropométricas, hemodinâmicas, composição corporal, e força máxima estática e dinâmica. Para a análise dos dados, utilizou-se ANOVA para medidas repetidas (grupo $\mathrm{x}$ tempo). Quando verificada diferença no momento inicial entre os grupos, a análise de covariância (ANCOVA) foi aplicada, utilizando como co-variável a linha de base. O post hoc de Scheffé foi aplicado para localizar as diferenças significantes. Os resultados mostraram que houve redução significante na somatória das nove dobras $(-14,80 \%)$, massa gorda $(-15,33 \%)$, no percentual de gordura $(-13,58 \%)$, aumento significante na força muscular (supino reto $+14,48 \%$; leg press $+13,21 \%)$ e pico de velocidade no teste de esteira $(+14,17 \%)$ após o treinamento. Tais resultados sugerem que o TC é eficaz para a melhora da composição corporal, força muscular, e aptidão aeróbia de homens de meia-idade saudáveis e sedentários.

Palavras-Chave: Composição corporal; Força muscular; Aptidão aeróbia; Treinamento de força; Treinamento aeróbio. 


\title{
MORPHO-FUNCTIONAL ADAPTATIONS AFTER 12 WEEKS OF CONCURRENT TRAINING ON MIDDLE-AGED MEN
}

\begin{abstract}
The purpose of this study was to analyze the morpho-functional adaptations after 12 weeks of concurrent training (CT). Fifteen healthy, sedentary, middle-aged men (48.8 \pm 5.0 years) participated on this study. They were divided in: concurrent training group $(\mathrm{CT}, \mathrm{n}=8)$, who performed resistance training, followed by walking and running exercises; and control group (CG, n=7), which didn't performed any type of exercise during the experimental period. Evaluation data on anthropometric, hemodynamic, body composition, maximal strength static and dynamic, was made. To analyze the data the repeated measures ANOVA was applied (group $\mathrm{x}$ time). When baseline presented differences between groups, the ANCOVA was applied setting the baseline values as co-variable. The Sheffé post hoc test was applied to locate significant differences. The results showed a reduction in the sum of nine skinfolds $(-14.80 \%)$, fat mass ($5.33 \%)$, fat percentage $(-13.58 \%)$, increased muscle strength (bench press and leg $14.48 \%$ press $13.21 \%)$ and peak velocity in the treadmill test $(+14.17 \%)$ in the post-training. These results suggests that CT is efficient to improve variables related to health of sedentary middle-aged, acting on a global way, influencing positively body composition, muscular strength, and aerobic fitness of these individuals.
\end{abstract}

Key-words: Body composition; Muscle strength; Aerobic fitness; Strength training; Endurance training.

Conexões: revista da Faculdade de Educação Física da UNICAMP, Campinas, v. 10, n. 1, p. 1-19, jan./abr. 2012. 


\section{INTRODUÇÃO}

O envelhecimento é caracterizado por alterações funcionais e morfológicas, como redução da força e massa muscular, que são definidas coletivamente como sarcopenia. ${ }^{1}$ Após a sétima e oitava década de vida a contração voluntária máxima é diminuída em média 20\% a 40\% para homens e mulheres. ${ }^{2}$ Em adição, estes prejuízos são agravados quando associados ao sedentarismo, potencializando a probabilidade de desenvolvimento de doenças crônicodegenerativas. ${ }^{3}$ O American College of Sports Medicine recomenda a realização de exercícios para o desenvolvimento e manutenção cardiorrespiratória, da força muscular e flexibilidade para a melhoria da saúde de indivíduos adultos idosos e jovens. ${ }^{4,5,6}$

$\mathrm{O}$ treinamento de força $(\mathrm{TF})$ pode promover uma pequena melhora em componentes aeróbios, porém resulta em aumento da força muscular e massa magra, atividade das enzimas glicolíticas, armazenamento de adenosina trifosfato (ATP)/fosfocreatina, ${ }^{6,7,9}$ atua na prevenção à osteoporose, ${ }^{10,11}$ além de aumentar a estabilidade dinâmica e preservar a capacidade funcional. ${ }^{4}$ Em contraste, o treinamento aeróbio (TA) aumenta a quantidade de mitocôndrias, ${ }^{12}$ densidade capilar, mioglobina intramuscular, enzimas que participam do ciclo de Krebs e cadeia de transporte de elétrons, além da capacidade e potência aeróbia. ${ }^{13}$ Desta forma, com um trabalho específico de TA, observa-se redução, tanto da frequência cardíaca de repouso, como da frequência cardíaca em potências submáximas, associado ao aumento do consumo máximo de oxigênio. ${ }^{14}$

Diversos estudos demonstraram que a associação entre TA e TF (treinamento concorrente) pode trazer benefícios tanto em variáveis relacionadas a força muscular, bem com a aptidão cardiorrespiratória. ${ }^{15,16,17,18} \mathrm{Em}$ adição, o treinamento concorrente (TC) tem sido considerado como o ideal para a saúde de pessoas de meia-idade e idosos. ${ }^{4,19}$

Desta forma, o objetivo deste estudo foi verificar as adaptações morfofuncionais decorrentes de um programa de TC para homens de meia-idade. A hipótese do presente estudo é que o TC promova adaptações advindas do TF, como aumento da força e massa magra, e ainda melhora na aptidão aeróbia e diminuição de massa gorda, mais relacionadas ao TA.

Conexões: revista da Faculdade de Educação Física da UNICAMP, Campinas, v. 10, n. 1, p. 1-19, jan./abr. 2012. ISSN: $1983-9030$ 


\title{
METODOLOGIA
}

\begin{abstract}
Amostra
Quinze homens de meia idade (48,12 \pm 5,05 anos), que não praticavam exercícios físicos por seis meses, se voluntariaram a participar deste estudo. Foram separados em grupo controle (GC, n=7), que não realizou nenhum tipo de programa de exercícios físicos sistematizados; e o grupo que realizou treinamento concorrente $(\mathrm{TC}, \mathrm{n}=8)$. O presente estudo foi aprovado pelo Comitê de Ética em Pesquisa da Universidade Estadual de Campinas, de acordo com as normas da Resolução 196/96 do Conselho Nacional de Saúde sobre pesquisa envolvendo seres humanos. Todos os sujeitos, após serem convenientemente informados sobre a proposta do estudo e procedimentos os quais seriam submetidos, assinaram declaração de consentimento esclarecido.
\end{abstract}

Foram excluídos desta pesquisa os voluntários que apresentaram na avaliação clínica e/ou nos exames laboratoriais, qualquer patologia ou outros complicadores que pudessem ser fatores de risco à adesão para a prática regular da atividade física proposta, tais como: doença arterial coronariana, hipertensão arterial, diabetes mellitus, doença pulmonar obstrutiva crônica, doenças ósteo-articulares limitantes, ou que estivesse utilizando qualquer medicação que pudesse interferir nas respostas fisiológicas aos testes.

\section{Instrumentos e Procedimentos}

A massa corporal (MC) foi obtida em uma balança (Filizola, Brasil) com precisão de 0,1 kg e a estatura foi determinada em um estadiômetro de madeira com precisão de $0,1 \mathrm{~cm}$, de acordo com os procedimentos descritos por Gordon, Chumlea e Roche. ${ }^{20}$ A partir das medidas de MC e estatura foi calculado o índice de massa corporal (IMC) por meio do quociente MC/estatura, ${ }^{2}$ sendo a MC expressa em quilogramas (kg) e a estatura em metros (m).

A estimativa dos componentes da composição corporal foi estabelecida pelo método de espessura do tecido celular adiposo subcutâneo (DC) onde foram aferidas com adipômetro (Lange), as seguintes DC: tricipital, subescapular, suprailíaca, bicipital, peitoral, coxa, panturrilha média e abdominal. Todas as medidas foram feitas por um único avaliador, todas do lado direito do corpo,

Conexões: revista da Faculdade de Educação Física da UNICAMP, Campinas, v. 10, n. 1, p. 1-19, jan./abr. 2012. ISSN: 1983-9030 
antes e após período experimental. A gordura corporal relativa (\%gordura) foi calculada pela fórmula de Jackson e Pollock, ${ }^{21}$ a partir da estimativa da densidade corporal determinada pela equação de Siri, ${ }^{22}$ que utiliza a somatória de três DC: peitoral, abdominal e coxa. Também como indicador da adiposidade corporal foi utilizada a somatória das nove DC supracitadas.

A força muscular dinâmica foi mensurada por meio do teste de uma repetição máxima (1-RM), conforme Clarke, ${ }^{23}$ nos exercícios de supino reto e leg press. A força estática isométrica de tração lombar (TL) e força estática de preensão manual direita (PMD) e esquerda (PME) foi mensurada com auxilio de dinamômetros.

Para a análise da flexibilidade, foram realizados medidas por meio de um flexímetro (Code) seguindo as recomendações de Achour Jr. ${ }^{24}$ Foram utilizados movimentos na posição ortostática - flexão dos ombros e flexão do tronco -, na posição decúbito dorsal - flexão do quadril - e na posição de decúbito ventral - flexão do joelho. Com exceção dos movimentos de flexão de tronco, todas as medidas foram coletadas bilateralmente.

As avaliações hemodinâmicas na condição de repouso (posição supina) utilizaram o registro da freqüência cardíaca durante um período de 60 minutos, com o auxílio de um monitor de frequência cardíaca da marca Polar $^{\circledR}$, modelo S810.

A avaliação cardiorrespiratória foi realizada em esteira rolante (Gesan, RY4000), com protocolo proposto para sedentários por Catai et al. ${ }^{25}$ Para obtenção da aptidão aeróbia dos voluntários, foi utilizado como parâmetros os valores da velocidade pico e frequência cardíaca em repouso, final do teste, e após período de 1 minuto de recuperação.

O TC foi realizado com duração de 12 semanas, com frequência de três sessões semanais de 60 minutos, dividido em duas etapas de 30 minutos cada, em dias alternados. A primeira etapa do treinamento constituía primeiramente de alongamento e aquecimento, seguidos de exercícios de força (supino reto, rosca alternada, elevação lateral, tríceps testa, agachamento com barra livre e abdominal), onde foram realizadas três séries de 15 repetições com pausas de um minuto, aproximadamente, entre elas, com exceção dos abdominais (três séries de 30 repetições). As

Conexões: revista da Faculdade de Educação Física da UNICAMP, Campinas, v. 10, n. 1, p. 1-19, jan./abr. 2012. ISSN: 1983-9030 
cargas utilizadas foram compatíveis ao número de repetições máximas estipuladas para cada exercício, sendo aproximadamente 50-70\% de 1-RM (ACSM, 2009b). Todas as cargas foram semanalmente ajustadas respeitando a zona-alvo de repetições máximas estabelecidas inicialmente para cada exercício. Na segunda etapa realizou-se 30 minutos de exercícios de caminhada e corrida, com intensidade de 70 a $85 \%$ da frequêencia cardíaca pico obtida na exaustão física durante o teste na esteira rolante. Ao final da sessão eram realizados alongamento para os principais grupos musculares.

\section{Análise estatística}

Para análise de normalidade dos dados foi utilizado o teste Shapiro-Wilks. Na comparação entre os grupos e entre os momentos analisados, foi utilizada a análise de variância (ANOVA) para medidas repetidas. Quando verificada diferença no momento inicial entre os grupos, a análise de covariância (ANCOVA) foi aplicada, utilizando como co-variável a linha de base. O teste post hoc de Scheffé foi utilizado para a identificação das diferenças específicas nas variáveis em que os valores de $\mathrm{F}$ encontrados foram superiores ao critério de significância estatística estabelecido $(\mathrm{P}<0,05)$.

Foi realizada a análise do poder da amostra post hoc através do software G*Power 3.0.10 ${ }^{26}$ com um alpha de 0.05. Todas as análises do poder foram feitas utilizando o efeito do tamanho (diferença entre os valores de base e os valores pós treinamento) e o "pooled standard deviation" (considerando valores pré e pós treino) das principais variáveis dependentes.

\section{Resultados}

\section{Análise do poder}

Para todas as variáveis dependentes analisadas o poder estatístico post hoc variou de 0.95 a 1.00.

\section{Adaptações morfofuncionais}

A Tabela 1 mostra os efeitos do programa de treinamento concorrente sobre as variáveis antropométricas e da composição corporal após período experimental. Não foram verificadas mudanças significantes para a massa corporal, índice de massa corporal e massa magra em 
nenhum dos grupos analisados. Contudo, a soma das nove dobras cutâneas, a gordura relativa e a massa gorda, apresentaram reduções significantes no TC após 12 semanas $(\mathrm{p}<0,05)$.

Tabela 1 - Variáveis antropométricas e composição corporal antes (pré) e após (pós) 12 semanas de período experimental

\begin{tabular}{|c|c|c|c|}
\hline Variáveis & & TC & GC \\
\hline MC (kg) & $\begin{array}{l}\text { Pré } \\
\text { Pós } \\
\Delta \%\end{array}$ & $\begin{array}{l}75,86 \pm 14,44 \\
73,47 \pm 14,38 \\
-3,15\end{array}$ & $\begin{array}{l}89,80 \pm 12,28 \\
89,78 \pm 12,21 \\
-0,02\end{array}$ \\
\hline $\operatorname{IMC}\left(\mathrm{kg} / \mathrm{m}^{2}\right)$ & $\begin{array}{l}\text { Pré } \\
\text { Pós } \\
\Delta \%\end{array}$ & $\begin{array}{l}26,01 \pm 3,09 \\
25,23 \pm 3,24 \\
-2,99\end{array}$ & $\begin{array}{l}30,54 \pm 3,96 \\
30,50 \pm 3,60 \\
-0,13\end{array}$ \\
\hline$\sum 9 \mathrm{DC}$ & $\begin{array}{l}\text { Pré } \\
\text { Pós } \\
\Delta \%\end{array}$ & $\begin{array}{l}245,81 \pm 83,18 \\
209,43 \pm 71,73 * \\
-14,80\end{array}$ & $\begin{array}{l}347,28 \pm 48,84 \\
338,14 \pm 54,55 \\
-2,63\end{array}$ \\
\hline GR (\%) & $\begin{array}{l}\text { Pré } \\
\text { Pós } \\
\Delta \%\end{array}$ & $\begin{array}{l}30,41 \pm 8,03 \\
26,28 \pm 8,22 * \\
-13,58\end{array}$ & $\begin{array}{l}37,93 \pm 3,60 \\
37,49 \pm 4,16 \\
-1,16\end{array}$ \\
\hline MG (kg) & $\begin{array}{l}\text { Pré } \\
\text { Pós } \\
\Delta \%\end{array}$ & $\begin{array}{l}23,80 \pm 10,05 \\
20,15 \pm 9,94 * \\
-15,33\end{array}$ & $\begin{array}{l}34,14 \pm 6 \\
33,87 \pm 6,95 \\
-0,79\end{array}$ \\
\hline MM (kg) & $\begin{array}{l}\text { Pré } \\
\text { Pós } \\
\Delta \%\end{array}$ & $\begin{array}{l}52,05 \pm 6,28 \\
53,32 \pm 5,75 \\
+2,43\end{array}$ & $\begin{array}{l}55,66 \pm 7,78 \\
55,91 \pm 6,81 \\
+0,44\end{array}$ \\
\hline Cintura $(\mathrm{cm})$ & $\begin{array}{l}\text { Pré } \\
\text { Pós } \\
\Delta \%\end{array}$ & $\begin{array}{l}90,60 \pm 8,67 \\
90,97 \pm 9,82 \\
+0,40\end{array}$ & $\begin{array}{l}101,92 \pm 7,84 \\
101,28 \pm 7,43 \\
-0,62\end{array}$ \\
\hline
\end{tabular}

Média \pm DP.

* Diferença significante após período experimental $(\mathrm{p}<0,05)$.

Legenda: TC - treinamento concorrente; GC - grupo controle; $\Delta \%$ - percentual de modificação; MC - massa corporal; IMC - índice de massa corporal; $\sum 9 D C$ - soma das 9 dobras cutâneas; GR - gordura relativa; MG - massa gorda; MM - massa magra.

No que diz respeito à flexibilidade, houve redução significante no movimento de flexão de ombro direito para os voluntários que realizaram TC $(\mathrm{p}<0,05)$. Foi verificada redução significante também no movimento de flexão de joelho direito para o $\mathrm{GC}(\mathrm{p}<0,05)$ (Tabela 2).

Conexões: revista da Faculdade de Educação Física da UNICAMP, Campinas, v. 10, n. 1, p. 1-19, jan./abr. 2012. 
Tabela 2 - Indicadores de flexibilidade em diferentes articulações antes (pré) e após (pós) 12 semanas de período experimental

\begin{tabular}{|c|c|c|c|}
\hline Variáveis & & TC & GC \\
\hline FT $\left(^{\circ}\right)$ & $\begin{array}{l}\text { Pré } \\
\text { Pós } \\
\Delta \%\end{array}$ & $\begin{array}{l}104,25 \pm 19,92 \\
105,37 \pm 16,75 \\
+1,07\end{array}$ & $\begin{array}{l}95,14 \pm 15,51 \\
92,57 \pm 14,54 \\
-2,87\end{array}$ \\
\hline $\mathrm{FQD}\left({ }^{\circ}\right)$ & $\begin{array}{l}\text { Pré } \\
\text { Pós } \\
\Delta \%\end{array}$ & $\begin{array}{l}99,62 \pm 7,48 \\
104,62 \pm 7,13 \\
+5,01\end{array}$ & $\begin{array}{l}96,71 \pm 13,74 \\
97,57 \pm 11,09 \\
+0,88\end{array}$ \\
\hline $\mathrm{FQE}\left({ }^{\circ}\right)$ & $\begin{array}{l}\text { Pré } \\
\text { Pós } \\
\Delta \%\end{array}$ & $\begin{array}{l}102,25 \pm 4,89 \\
108,75 \pm 11,49 \\
+6,35\end{array}$ & $\begin{array}{l}93,28 \pm 15,40 \\
95,14 \pm 11,48 \\
+1,99\end{array}$ \\
\hline $\operatorname{FJD}\left(^{\circ}\right)$ & $\begin{array}{l}\text { Pré } \\
\text { Pós } \\
\Delta \%\end{array}$ & $\begin{array}{l}129,12 \pm 7,41 \\
130,37 \pm 4,81 \\
+0,96\end{array}$ & $\begin{array}{l}118,28 \pm 14,96 \\
112,28 \pm 11,98 * \\
-5,07\end{array}$ \\
\hline $\mathrm{FJE}\left({ }^{\circ}\right)$ & $\begin{array}{l}\text { Pré } \\
\text { Pós } \\
\Delta \%\end{array}$ & $\begin{array}{l}128,12 \pm 6,92 \\
128,62 \pm 6,99 \\
+0,39\end{array}$ & $\begin{array}{l}116,85 \pm 10,45 \\
113,42 \pm 10,16 \\
-2,93\end{array}$ \\
\hline $\mathrm{FOD}\left(^{\circ}\right)$ & $\begin{array}{l}\text { Pré } \\
\text { Pós } \\
\Delta \%\end{array}$ & $\begin{array}{l}147,87 \pm 9,63 \\
135,00 \pm 6,89^{*} \\
-8,70\end{array}$ & $\begin{array}{l}150,14 \pm 10,88 \\
152,28 \pm 14,15 \\
+1,42\end{array}$ \\
\hline $\operatorname{FOE}\left({ }^{\circ}\right)$ & $\begin{array}{l}\text { Pré } \\
\text { Pós } \\
\Delta \%\end{array}$ & $\begin{array}{l}148,25 \pm 9,54 \\
139,12 \pm 8,20 \\
-6,15\end{array}$ & $\begin{array}{l}145,57 \pm 11,50 \\
151,85 \pm 11,14 \\
+4,31\end{array}$ \\
\hline
\end{tabular}

Média \pm DP.

* Diferença significante após período experimental $(\mathrm{p}<0,05)$.

Legenda: TC - treinamento concorrente; GC - grupo controle; $\Delta \%$ - percentual de modificação; FT flexão de tronco; FQD - flexão de quadril direito; FQE - flexão de quadril esquerdo; FJD flexão de joelho direito; FJE - flexão de joelho esquerdo; FOD - flexão de ombro direito; FOE - flexão de ombro esquerdo.

Nos testes de força estática de preensão manual, nenhum dos dois grupos apresentou modificações significantes após a realização do experimento. Em relação ao teste de 1-RM, houve aumento significante $(\mathrm{p}<0,05)$ na força máxima para os exercícios de leg press e supino reto no TC (Tabela 3).

Conexões: revista da Faculdade de Educação Física da UNICAMP, Campinas, v. 10, n. 1, p. 1-19, jan./abr. 2012. 
Tabela 3 - Indicadores de força estática e dinâmica antes (pré) e após (pós) 12 semanas de período experimental

\begin{tabular}{llll}
\hline Variáveis & & \multicolumn{1}{c}{ TC } & \multicolumn{1}{c}{ GC } \\
\hline PMD $(\mathrm{kg})$ & Pré & $37,75 \pm 4,28$ & $45,50 \pm 12,08$ \\
& Pós & $39,37 \pm 4,90$ & $46,07 \pm 11,96$ \\
& $\Delta \%$ & $+4,29$ & $+1,21$ \\
& & & \\
PME $(\mathrm{kg})$ & Pré & $38,37 \pm 7,43$ & $45,00 \pm 5,13$ \\
& Pós & $42,12 \pm 8,27$ & $45,00 \pm 4,31$ \\
& $\Delta \%$ & $+9,77$ & 0,00 \\
& & & \\
TL $(\mathrm{kg})$ & Pré & $99 \pm 24,27$ & $117,57 \pm 15,13$ \\
& Pós & $102,87 \pm 16,59$ & $117 \pm 25,74$ \\
& $\Delta \%$ & $+3,90$ & $-0,48$ \\
$1-\mathrm{RM} \mathrm{SR}(\mathrm{kg})$ & Pré & $59,50 \pm 14,96$ & $77,21 \pm 8,66$ \\
& Pós & $68,12 \pm 14,24 *$ & $84,28 \pm 16,11$ \\
& $\Delta \%$ & $+14,48$ & $+9,15$ \\
& & & $162,14 \pm 10,24$ \\
$1-\mathrm{RM} \mathrm{LP}(\mathrm{kg})$ & Pré & $145,62 \pm 25$ & $171,42 \pm 7,21$ \\
& Pós & $164,87 \pm 15,36^{*}$ & $+5,72$ \\
\hline
\end{tabular}

Média \pm DP.

* Diferença significante após período experimental $(\mathrm{p}<0,05)$.

Legenda: TC - treinamento concorrente; GC - grupo controle; $\Delta \%$ - percentual de modificação; PMD - força estática de preensão manual direita; PME - força estática de preensão manual esquerda; TL - força de tração lombar; 1-RM SR - uma repetição máxima no exercício supino reto; 1-RM LP - uma repetição máxima no exercício leg press.

Dentre as variáveis cardiorrespiratórias e hemodinâmicas, houve alteração significante apenas na velocidade pico, aumentando após 12 semanas de TC (p<0,05) (Tabela 4).

Conexões: revista da Faculdade de Educação Física da UNICAMP, Campinas, v. 10, n. 1, p. 1-19, jan./abr. 2012. 
Tabela 4 - Variáveis cardiorrespiratórias antes (pré) e após (pós) 12 semanas de período experimental

\begin{tabular}{llll}
\hline Variáveis & & \multicolumn{1}{c}{ TC } & \multicolumn{1}{c}{ GC } \\
\hline FCsup $(\mathrm{bpm})$ & Pré & $65,75 \pm 8,86$ & $82,28 \pm 12,42$ \\
& Pós & $66,62 \pm 6,44$ & $75,57 \pm 19,87$ \\
& $\Delta \%$ & $+1,32$ & $-8,15$ \\
FCpico $(\mathrm{bpm})$ & & & \\
& Pré & $171,62 \pm 19,26$ & $175,85 \pm 11,42$ \\
& Pós & $174,50 \pm 15,88$ & $151,57 \pm 37,41$ \\
& $\Delta \%$ & $+1,67$ & $-13,80$ \\
FC após $1 \mathrm{~min} .(\mathrm{bpm})$ & Pré & $105,25 \pm 8,99$ & $146,57 \pm 7,98$ \\
& Pós & $114,00 \pm 9,04$ & $133,14 \pm 29,39$ \\
& $\Delta \%$ & $+8,31$ & $-9,16$ \\
Vel.pico $(\mathrm{km} / \mathrm{h})$ & & & $8,45 \pm 0,87$ \\
& Pré & $10,51 \pm 2,01$ & $8,80 \pm 0,88$ \\
& Pós & $12,00 \pm 2,04 *$ & $+4,14$ \\
\hline
\end{tabular}

Média \pm DP.

* Diferença significante após período experimental $(\mathrm{p}<0,05)$.

Legenda: TC - treinamento concorrente; GC - grupo controle; $\Delta \%$ - percentagem de modificação; FCsup - frequência cardíaca supina; FCpico - frequência cardíaca pico; FC após 1 min. frequência cardíaca após um minuto do término do teste de esforço; Vel.pico - velocidade pico.

\section{DISCUSSÃO}

Os achados do presente estudo sugerem que o TC promove melhoras significativas na aptidão aeróbia, na força muscular e composição corporal. Estes dados corroboram com os encontrados em alguns estudos que também avaliaram os efeitos do TC. ${ }^{15,16,17,27,28}$

Em relação à composição corporal, o presente estudo verificou uma redução na gordura corporal após 12 semanas de TC, porém sem aumento significante na massa magra. Esse resultado pode estar associado às dificuldades da manutenção e ganho de massa muscular após os 35 anos de idade, especialmente em indivíduos pouco ativos, ${ }^{29,30}$ ou até mesmo, as adaptações concorrentes ocasionadas pelos diferentes metodologias de treinamento. ${ }^{31}$ 
Uma das possíveis explicações do ocorrido nesse estudo em relação à massa magra pode estar relacionada às diferentes respostas hormonais ao treinamento físico. $\mathrm{O}$ TF aumenta as concentrações de testosterona ${ }^{32,33}$ o que não é observado em relação à concentração de cortisol. $\mathrm{O}$ cortisol apresenta valores maiores a partir da influência do TA, sendo este, um hormônio catabólico, que possui um efeito prejudicial ao ganho de massa muscular, ao reduzir a síntese protéica e aumentar a degradação de proteína. ${ }^{34}$ Contudo, na elaboração do TC no presente estudo houve a preocupação que a duração total da sessão de TF fosse a mesma do TA, e que a soma de ambas não ultrapassasse 60 minutos, visando minimizar o aumento das concentrações de cortisol.

Tendo em vista as recomendações do $\mathrm{ACSM},{ }^{6}$ o programa proposto se enquadra na prescrição para resistência muscular localizada (1-3 séries de 10-15 repetições), o que aparentemente não favorece de maneira significativa a hipertrofia muscular. Associado a este fato, o período de 12 semanas pareceu não ser significativo para o aumento de massa magra. Estudos tem demonstrado ganhos de massa magra em períodos de treinamento maiores (21 semanas), ${ }^{35}$ assim como também em períodos menores (10 semanas). ${ }^{16}$ Essas respostas sugerem que as diferenças nos programas de treinamento (volume $\mathrm{x}$ intensidade), parecem ser o ponto crucial em relação à otimização do TC.

As reduções significantes na gordura corporal encontradas neste estudo são corroboradas com outros estudos. ${ }^{17}$ Hunter, Demment e Miller, ${ }^{17}$ compararam TF e TC por período de 12 semanas e observaram maiores reduções na gordura relativa para o TC em relação ao TF $(2,6 \%$ e 1,6\%, respectivamente). Apesar de apresentar a mesma duração do TC em relação ao estudo de Hunter, Demment e Miller, ${ }^{17}$ maiores reduções no percentual de gordura (13,58\%), no somatório de nove dobras $(14,80 \%)$ e na massa gorda $(\mathrm{MG})(15,33 \%)$ foram observadas no presente trabalho.

No que diz respeito à circunferência de cintura, um ponto positivo é que o TC não teve seus valores aumentados. A circunferência de cintura é um indicativo de gordura corporal que merece atenção, uma vez que está relacionada à saúde, apresentando estreita relação com a resistência à insulina e doenças cardiovasculares. ${ }^{36,37}$

Conexões: revista da Faculdade de Educação Física da UNICAMP, Campinas, v. 10, n. 1, p. 1-19, jan./abr. 2012. ISSN: 1983-9030 
Embora não tenha ocorrido aumento significante na massa magra, o presente estudo demonstrou melhoras na força dinâmica avaliada pelos testes de 1-RM para o exercício supino reto $(14,48)$ e leg press $(13,21 \%)$. O ganho de força sem aumento da massa magra tem sido bem documentado em indivíduos adultos, de meia-idade e idosos, observados principalmente nas semanas iniciais de treinamento, sendo atribuído ao aumento da ativação das unidades motoras na musculatura envolvida. ${ }^{38,39,40,41,42,43}$ A adaptação neural também esta associada a uma redução da coativação do músculo antagonista, contribuindo ainda mais para o aumento da força. ${ }^{39}$

Häkkinen et al., ${ }^{35}$ observaram uma grande redução da coativação do músculo bíceps femoral analisado por EMG durante o TC, além de uma maior ativação do músculo quadríceps após 7, 14 e 21 semanas de treinamento, demonstrando que os ganhos de força não sofreram efeitos adversos como demonstrados em outros estudos. ${ }^{27,44,45,46}$ Sendo assim o TC parece nem sempre influenciar negativamente o desenvolvimento da força muscular. ${ }^{15,28,35,47}$ Já a força estática pode ter sido influenciada pela especificidade do treinamento, uma vez que não sofreu alterações significantes.

Outro fator que pode comprometer o desenvolvimento da força muscular pode ser a depleção das reservas de glicogênio, principalmente quando as sessões de TA são alternadas com as de TF e sendo executadas diariamente, ou até mesmo duas sessões no mesmo dia. ${ }^{48}$ No presente estudo a sessão de TC não teve longa duração, e ainda o intervalo entre elas foi de 48 horas o que permitiu uma melhor recuperação dos voluntários. Igualmente vale lembrar que a TF foi realizado antes do TA.

Poucos são os estudos que retratam a influência do TC sobre a flexibilidade de indivíduos de meia-idade. O envelhecimento, associado ao sedentarismo, é capaz de promover alterações fisiológicas deletérias à saúde, como a consequente redução da flexibilidade. ${ }^{49}$ Os achados aqui apresentados permitem destacar a importância do treinamento proposto para indivíduos de meiaidade, demonstrando que o TC não influência a flexibilidade de maneira negativa. Resultados similares em relação à flexibilidade foram observados no estudo de Barbosa, Santarém, Jacob Filho e Marucci ${ }^{50}$ que estudaram o efeito do TF na flexibilidade de jovens e Cyrino et al. ${ }^{51}$ que

Conexões: revista da Faculdade de Educação Física da UNICAMP, Campinas, v. 10, n. 1, p. 1-19, jan./abr. 2012. ISSN: $1983-9030$ 
estudou mulheres idosas. Vale ressaltar que nesses estudos, não foram realizados exercícios de alongamento antes e após as sessões de treinamento.

Nas variáveis relacionadas aos aspectos hemodinâmicos, apesar da FCsup, FCpico e FC após 1 min., não mostrarem alterações significativas após o período experimental, houve aumento significante na velocidade pico $(14,17 \%)$. Campos et al., ${ }^{7}$ verificaram que o TF com altas repetições pode proporcionar um aumento na velocidade pico em testes contínuos. Segundo Millet, Jaouen, Borani, e Candau, ${ }^{52}$ isto é caracterizado como economia de movimento e pode acontecer mesmo quando o TA é concorrente ao TF de baixas repetições e cargas elevadas.

É possível que o TC, proposto neste estudo, seja mesmo o mais adequado para este tipo de população (i.e., indivíduos no início do processo de envelhecimento e sedentários) uma vez que igualar o tempo da sessão ou frequência semanal do TC ao TF seja a melhor forma de otimizar essas adaptações. ${ }^{28,53}$

De acordo com os dados apresentados, podemos concluir que o TC proposto, melhorou a aptidão física de homens de meia-idade, mostrado pela redução na gordura corporal e aumento na força muscular. Também apresentou evidências que o TC, não reduz a massa magra, bem como a flexibilidade. Desta forma, fica evidenciado a importância deste método de treinamento para minimizar as alterações morfofuncionais decorrentes do envelhecimento.

Uma análise mais fidedigna de composição corporal (e.g. análise por Dual-Enery X-ray Absorptiometry - DEXA) e aptidão aeróbia $\left(\mathrm{VO}_{2}\right)$ poderiam contribuir ainda mais com as discussões do presente estudo. Adicionalmente, seria interessante a comparação das adaptações isoladas do TF e TA com os resultados encontrados pelo TC. Desta forma, a realização de futuros estudos é necessária para consolidar os conhecimentos que envolvem o TC, abarcando diversas populações e metodologias de treinamento, para esclarecer os resultados positivos e as interferências da combinação do treinamento aeróbio e ao treinamento força.

Conexões: revista da Faculdade de Educação Física da UNICAMP, Campinas, v. 10, n. 1, p. 1-19, jan./abr. 2012. ISSN: 1983-9030 


\section{REFERÊNCIAS}

${ }^{1}$ DUTTA, C.; HADLEY, E. C. The significance of sarcopenia in old age. Journal of Gerontology Series: a biological science and medical science, v. 50: p. 1-4, 1995.

${ }^{2}$ DOHERT, T. J. Aging and sarcopenia. Journal of Applied Physiology, v. 95, p. 1717-27, 2003.

${ }^{3}$ STEWART, K. J. et al. Exercise and risk factors associated with metabolic syndrome in older adults. American Journal of Preventive Medicine, v. 28, n. 1, p. 9-18, 2005.

${ }^{4}$ AMERICAN COLLEGE OF SPORTS MEDICINE. The recommended quantity and quality of exercise for developing and maintaining cardiorespiratory and muscular fitness, and flexibility in healthy adults. Medicine Science of Sports Exercise, v. 30, n. 6, p. 975-991, 1998.

${ }^{5}$ AMERICAN COLLEGE OF SPORTS MEDICINE. Exercise and physical activity for older adults. Medicine Science of Sports Exercise, v. 41, n. 7, p. 1510-1530, 2009a.

${ }^{6}$ AMERICAN COLLEGE OF SPORTS MEDICINE. Progression models in resistance training for healthy adults. Medicine Science of Sports Exercise, p. 687-708, 2009 b.

${ }^{7}$ CAMPOS, G. E. et al. Muscular adaptations in response to three resistance training regimes: specificity of repetition maximum training zones. European Journal of Applied Physiology, v. 88 , n. $1 / 2$, p. 50-60, 2002.

${ }^{8}$ FLETCHER, G. F. et al. Exercise standards: a statement for healthcare professionals from the American Heart Association. Circulation, v. 91, p. 580-615, 1995.

${ }^{9}$ Pollock, M. L. et al. (2000). Resistance exercise in individuals with and without cardiovascular disease: benefits, rationale, safety, and prescription. Circulation, v. 101, p. 828-833, 2000. 
${ }^{10}$ GUTIN, B.; KASPER, M. J. Can exercise play a role in osteoporosis prevention? a review. Osteoporosis International, n. 2, p. 55-69, 1992.

${ }^{11}$ LAYNE, J. E.; NELSON, M. E. The effect of progressive resistance training on bone density: a review. Medicine Science Sports Exercise, v. 31, p. 25-30, 1999.

${ }^{12}$ LUMINI, J. A. et al. Beneficial effects of exercise on muscle mitochondrial function in diabetes mellitus. Sports Medicine, v. 38, n. 9, p. 735-750, 2008.

${ }^{13}$ TAYLOR, A. W.; BACHMAN, L. The effects of endurance training on muscle fibre types and enzyme activities. Canadian Journal of Applied Physiology, v. 24, n. 1, p. 41-53, 1999.

${ }^{14}$ CHACON-MIKAHIL, M. P. T. et al. Cardiorespiratory adaptations induced by aerobic training in middle-aged men: the importance of a decrease in sympathetic stimulation for the contribution of dynamic exercise tachycardia. Brazilian Journal of Medicine Biology Resistance, v. 31, $\mathrm{n}$. 5, p. 705-712, 1998.

${ }^{15}$ BALABINIS, C. P. et al. Early phase changes by concurrent endurance and strength training. Journal of Strength and Conditioning Research, v. 17, n. 2, p. 393-401, 2003.

${ }^{16}$ DOLEZAL, B. A.; POTTEIGER, J. A. Concurrent resistance and endurance training influence basal metabolic rate in nondieting individuals. Journal of Applied Physiology, v. 85, n. 2, p. 695-700, 1998.

${ }^{17}$ HUNTER, G. ; DEMMENT, R. ; MILLER, D. Development of strength and maximum oxygen uptake during simultaneous training for strength and endurance. Journal of Sports Medicine and Physical Fitness, v. 27, n. 3, p. 269-275, 1987.

${ }^{18}$ KARAVIRTA, L. et al. Heart rate dynamics after combined endurance and strength training in older men. Medicine Science Sports Exercise, v. 41, p. 1436-1443, 2009.

Conexões: revista da Faculdade de Educação Física da UNICAMP, Campinas, v. 10, n. 1, p. 1-19, jan./abr. 2012. ISSN: 1983-9030 
${ }^{19}$ HURLEY, B. F.; HAGBERG, J. M. Optimizing health in older persons: aerobic or strength training? Exercise Sports Science Review, v. 26, p. 61-89, 1998.

${ }^{20}$ GORDON, C. C. ; CHUMLEA, W. C. ; ROCHE, A. F. (1988). Stature, recumbent length, and weight. In: LOHMAN T.G.; ROCHE A. F.; MARTORELL R. (Ed.). Anthropometric standardization reference manual. Champaign: Human Kinetics Books, 1988. p. 3-8.

${ }^{21}$ JACKSON, A. S.;POLLOCK, M. L. Generalized equations for predicting body density of men. Brazilian Journal of Nutrition, v. 40, n. 3, p. 497-504, 1978.

${ }^{22}$ SIRI, W. E. (1961). Body composition from fluid spaces and density: Analysis of methods. In: J. BROZEK, J.; HENSCHEL, A. (Ed.). Techniques for measuring body composition. Washington: National Academic of Science, 1961. p. 223-244.

${ }^{23}$ CLARKE, D. H. Adaptations in strength and muscular endurance resulting from exercise. In: Wilmore, J. H. (Ed.). Exercise and sports sciences reviews. New York: Academic Press, 1973. p. 73-102.

${ }^{24}$ ACHOUR JR., A. Manual de instruções: fleximeter, avaliando a flexibilidade. Londrina: Midiograf, 1997.

${ }^{25}$ CATAI, A. M. et al. Cardiorrespiratory responses during dynamic exercise using different ergometers in sedentary young men. FASEB Journal, v.10, n. 3, p. 375, 1996.

${ }^{26}$ FAUL, F. et al. G*Power 3: a flexible statistical power analysis program for the social, behavioral, and biomedical sciences. Behavior Research Methods, v. 39, n. 2, p. 175-191, 2007.

${ }^{27}$ CADORE, E. L. Physiological effects of concurrent training in elderly men. International Journal of Sports Medicine, 2010. DOI http://dx.doi.org/10.1055/s-030-1261895 
${ }^{28}$ IZQUIERDO, M. Once weekly combined resistance and cardiovascular training healthy older men. Medicine and Science in Sports and Exercise, v. 36, n. 3, p. 435-443, 2004.

${ }^{29}$ BORST, S. E. Interventions for sarcopenia and muscle weakness in older people. Age and Ageing, v. 33, n. 6, p. 548-555, 2004.

${ }^{30}$ TAAFFE, D. R. Sarcopenia: exercise as a treatment strategy. Australian Family Physician, v. 35, n. 3, p. 130-133, 2006.

${ }^{31}$ TANAKA, H.; SWENSEN, T. Impact of resistance training on endurance performance a new form of cross-training? Sports Medicine, v. 25, p.191-200, 1998.

${ }^{32}$ KRAEMER, W. J; RATAMESS, N. A. Hormonal responses and adaptations to resistance exercise and training. Sports Medicine, v. 35, n. 4, p. 339-361, 2005.

${ }^{33}$ VOLEK, J. S. et al. Testosterone and cortisol in relationship to dietary nutrients and resistance exercise. Journal of Applied Physiology, v. 82, n. 1, p. 49-54, 1997.

${ }^{34}$ DESCHENES, M. R.; KRAEMER, W. J. Performance and physiologic adaptations to resistance training. American Journal of Physiology Medicine Rehabilitation, v. 81, suppl., p. S3-S16, 2002.

${ }^{35} \mathrm{HÄKKINEN}$, K. et al. Neuromuscular adaptations during concurrent strength and endurance training versus strength training. European Journal of Applied Physiology, v. 89, p. 42-52, 2003.

${ }^{36}$ KAHN, S. E.; HULL, R. L.; UTZSCHNEDER, K. M. Mechanisms linking obesity to insulin resistance and type 2 diabetes. Nature, v. 444, n. 7121, p. 840-846, 2006.

${ }^{37} \mathrm{KOHRT}$, W. M. et al. Insulin resistance in aging is related to abdominal obesity. Diabetes, v. 42, n. 2, p. 273-281, 1993.

Conexões: revista da Faculdade de Educação Física da UNICAMP, Campinas, v. 10, n. 1, p. 1-19, jan./abr. 2012. 
${ }^{38}$ HÄKKINEN, K.; KOMI, P. V. Electromyographic changes during strength training and detraining. Medicine Science Sports Exercise, v. 15, p. 455-460, 1983.

${ }^{39} \mathrm{HÄKKINEN}, \mathrm{K}$. et al. Changes in agonist-antagonist EMG, muscle CSA and force during strength training in middle-aged and older people. Journal Applied Physiology, v. 84, p.1341$1349,1998$.

${ }^{40} \mathrm{HÄKKINEN}$, K. et al. Selective muscle hypertrophy, changes in EMG and force, and serum hormones during strength training in older women. Journal of Applied Physiology, v. 91, p. 569-580, 2001b.

${ }^{41} \mathrm{HÄKKINEN,} \mathrm{K}$. et al. Changes in electromyographic activity, muscle fibre and force production characteristics during heavy resistance/power strength training in middle-aged and older men and women. Acta Physiology Scandinavian, v.171, p. 51-62, 2001a.

${ }^{42}$ MORITANI, T.; DEVRIES, H. Neural factors versus hypertrophy in the time course of muscle strength gain. American Journal of Physical Medicine, v. 58, n. 3, p. 115-129, 1979.

${ }^{43}$ MORITANI, T.; DEVRIES, H. A. Potential for gross muscle hypertrophy in older men. Journal Gerontology, v. 35, p. 672-682, 1980.

${ }^{44}$ KRAEMER, W. J. et al. Compatibility of high-intensity strength and endurance training on hormonal and skeletal muscle adaptations. Journal of Applied Physiology, v. 78, p. 976-989, 1995.

${ }^{45}$ MCCARTHY, J. P. et al Compatibility of adaptive responses with combining strength and endurance training. Medicine Science Sports Exercise, v. 27, p. 429-436, 1995.

${ }^{46}$ MCCARTHY, J. P.; POZNIAK, M. A.; AGRE, J. C. Neuromuscular adaptations to concurrent strength and endurance training. Medicine Science Sports Exercise, v. 34, 511-519, 2002.

Conexões: revista da Faculdade de Educação Física da UNICAMP, Campinas, v. 10, n. 1, p. 1-19, jan./abr. 2012. ISSN: 1983-9030 
${ }^{47}$ FLECK, S. J.; MATTIE, C.; MARTENSEN III, H. Effect of resistance and aerobic training on regional body composition in previously recreationally trained middle-aged women. Applied Physiology Nutrition Metabolism, v. 31, n. 3, p. 261-270, 2006.

${ }^{48}$ NADER, G. A. Concurrent strength and endurance training: from molecules to man. Medicine Science Sports Exercise, v. 38, n. 11, p. 1965-1970, 2006.

${ }^{49}$ HASS, C. J. et al. Concurrent improvements in cardiorespiratory and muscle fitness in response to total body recumbent stepping in humans. European Journal of Applied Physiology, v. 85, n.1/2, 157-163, 2001.

${ }^{50}$ BARBOSA, A. R. et al. Effects of resistance training on the sit-and-reach test in elderly women. Journal of Strength and Conditioning Research, v.16, n. 1, p. 14-18, 2002.

${ }^{51}$ CYRINO, E. S. et al. Comportamento da flexibilidade após 10 semanas de treinamento com pesos. Revista Brasileira de Medicina do Esporte, v. 10, n. 4, p. 233-237, 2004.

${ }^{52}$ MILLET, G. P. et al. Effect of concurrent endurance and strength training on running economy and VO 2 kinetics. Medicine Science Sports Exercise, v. 34, p. 1351-1359, 2002.

${ }^{53}$ WOOD, R. H. et al. Concurrent cardiovascular and resistance training in healthy older adults. Medicine Science Sports Exercise, v. 33, n. 10, p. 1751-1758, 2001.

Nota do autor

Laboratório de Fisiologia do Exercício - FISEX, Faculdade de Educação Física Universidade Estadual de Campinas.

Endereço para correspondência: Mara Patrícia Traina Chacon-Mikahil, Faculdade de Educação Física/Universidade Estadual de Campinas - UNICAMP, Av. Érico Veríssimo, 701, Caixa Postal 6134, CEP: 13083-851 Campinas, Brasil. E-mail: marapatricia@fef.unicamp.br 